\title{
The assessment of the body fat percentage by skinfold thickness measurements in childhood and young adolescence
}

\author{
BY PAUL DEURENBERG, JOHN J. L. PIETERS* \\ AND JOSEPH G. A. J. HAUTVAST \\ Department of Human Nutrition, Agricultural University, Bomenweg 2, NL 6703 HD Wageningen, \\ The Netherlands
}

(Received 7 March 1989 - Accepted 14 October 1989)

\begin{abstract}
The relationship of skinfold thicknesses to body density and of skinfold thicknesses to densitometrically determined body fat was evaluated in a group of 378 boys and girls, aged 7-20 years. According to their maturation level, they were divided into a prepubertal, a pubertal and a post-pubertal group. In each maturation group boys were older, had higher body-weights and body heights, higher body densities, lower percentage body fat, higher waist : hips ratios and higher trunk :total skinfolds ratios than girls. Body density in each maturation level could be quite precisely predicted by skinfold thicknesses. In prepubertal and pubertal boys and girls but not in post-pubertal boys and girls, age was also an important predicting variable for body density. The assessment of percentage body fat from skinfold thicknesses had a prediction error of 3-5\%, which was highest in the prepubertal children. The prediction error is comparable to the prediction error of percentage body fat from skinfold thicknesses in adults, as reported in the literature. Only in post-pubertal boys and girls was the waist:hip ratio correlated with measures of body fatness. Moreover, only in the post-pubertal boys and in the pubertal and post-pubertal girls was the waist:hips ratio correlated with another measure of body fat distribution, the trunk:total skinfold ratio. The relative amount of internal body fat was found to be higher in the younger maturation groups. It is concluded that at younger ages the waist :hips ratio is a poor indicator of body fat distribution.
\end{abstract}

Skinfold thickness: Body fat: Maturation status: Children

The assessment of percentage body fat in children is not an easy task, for several reasons. First, the chemical composition of the fat-free mass in children is different from that in the adults in whom carcass analyses were performed (Mitchell et al. 1945; Widdowson et al. 1951; Forbes et al. 1953, 1956), and second, the chemical composition changes during maturation (Lohman et al. 1984; Boileau et al. 1981, 1984, 1985; Lohman, 1986; Forbes, 1987; Weststrate \& Deurenberg, 1989). Therefore the assumptions used for the calculation of body composition in adults from (for example) body density, ${ }^{40} \mathrm{~K}$ determinations or dilution techniques cannot be applied to growing children. Several authors (Fomon et al. 1982; Lohman, 1986; Slaughter et al. 1988; Weststrate \& Deurenberg, 1989) have therefore proposed new assumptions for the calculation of body fat percentage from body density values in children and young adolescents.

Apart from these theoretical considerations, another problem in the determination of body composition in children is that the cooperation necessary for some measurements, for example underwater weighing, is often difficult to obtain, especially in very young children. An easy method of assessing the percentage body fat in children would be measurement of the subcutaneous fat layer, i.e. skinfold thickness. However, due to a changing (subcutaneous) fat pattern during maturation (Frisancho, 1981; Forbes, 1987; Weststrate et al. 1989), the relation between skinfold thicknesses and body density (and, hence, total

* Present address: Chief Medical Office, PO Box 5406, NL 2280 AK Rijswijk, The Netherlands. 
body fat) could be strongly dependent on (biological) age, which would hamper the assessment of percentage body fat by skinfold thicknesses.

The aim of the present study was to determine the relationship between skinfold thicknesses and body density and percentage body fat in boys and girls from prepuberty to young adulthood.

\section{SUBJECTS AND METHODS}

A total of 208 girls and 170 boys, aged 7-20 years, participated in the study. They were recruited from primary and secondary schools. The aim of the study and the kind of measurements were explained to the participants during a school visit, after which the measurements were performed at the Department of Human Nutrition.

Body-weight was measured to the nearest $0.05 \mathrm{~kg}$ (ED-60T; Berkel, Rotterdam) in swimming clothes. Body height was measured with a microtoise to the nearest $1 \mathrm{~mm}$. Skinfold thicknesses were measured in triplicate at the left side of the body to the nearest $0.1 \mathrm{~mm}$ with Harpenden skinfold calipers (Holtain Ltd, Bryberian, UK) at the following sites: (1) triceps, halfway between the acromion process and the olecranon process; (2) biceps, at the same level as the triceps skinfold, directly above the centre of the cubital fossa; (3) subscapular, about $20 \mathrm{~mm}$ below the tip of the scapula, at an angle of $45^{\circ}$ to the lateral side of the body; (4) supra-iliac, about $20 \mathrm{~mm}$ above the iliac crest, in the axillary line. To correct for a skewed distribution of the measured values, the skinfolds were transformed $\left(\log _{10}\right)$ before statistical analysis. Waist circumference was measured, midway between the lower rib margin and the iliac crest, with a plastic tape to the nearest $1 \mathrm{~mm}$. Hip circumference (to the nearest $1 \mathrm{~mm}$ ) was measured at the level of the greater trochanter.

Body density was measured by means of underwater weighing to the nearest $0.05 \mathrm{~kg}$ (3826 MP 81; Sartorius, Göttingen, Germany) in duplicate with simultaneous determination of the lung volume by helium dilution (Spiro-Junior, Jaeger GMBH, Würtzburg, Germany).

The reproducibility of the measurements is about $0.0006 \mathrm{~kg} / 1(0.3 \%$ body fat $)$ in adults and about $0.0048 \mathrm{~kg} / \mathrm{l}(2.5 \%$ body fat) in young children (Deurenberg et al. $1989 \mathrm{~b})$. For both boys and girls percentage body fat was calculated from body density using the formula

$$
\text { body fat }(\%)=\frac{(562-4 \cdot 2(\text { age }-2))}{\text { body density }}-(525-4 \cdot 7(\text { age }-2)) \text {, }
$$

where age is expressed in years (Weststrate \& Deurenberg, 1989). In this formula it is assumed that the density of the fat-free mass slowly increases with age, from $1.080 \mathrm{~kg} / 1 \mathrm{at}$ 7 years (Fomon et al. 1982) to $1 \cdot 100 \mathrm{~kg} / 1$ at 18 years (Siri, 1961) in both sexes. Maturation status was assessed by the classification described by Tanner (1962) and by van Wieringen et al. (1968), which is based on the extent of hair covering of the pubertal region, (six stages: $\left.\mathrm{p}_{1}-\mathrm{p}_{6}\right)$. The study population was divided into a prepubertal $\left(\mathrm{p}_{1}-\mathrm{p}_{2}\right)$, a pubertal $\left(\mathrm{p}_{3}-\mathrm{p}_{4}\right)$ and a post-pubertal $\left(\mathrm{p}_{5}-\mathrm{p}_{6}\right)$ group.

Tables 1 and 2 list some of the physical characteristics of the male and female subgroups respectively.

Simple linear regression and stepwise multiple regression analyses were performed using the SPSS-PC program (SPSS, 1988). Differences in variables were tested by analysis of variance or paired Student's $t$ tests. Values are expressed as means with their standard errors. 
Table 1. Physical characteristics of prepubertal, pubertal and post-pubertal boys

(Mean values with their standard errors; no. of boys in parentheses)

\begin{tabular}{|c|c|c|c|c|c|c|}
\hline & \multicolumn{2}{|c|}{$\begin{array}{l}\text { Prepubertal } \\
\quad \text { (114) }\end{array}$} & \multicolumn{2}{|c|}{$\begin{array}{l}\text { Pubertal } \\
\text { (35) }\end{array}$} & \multicolumn{2}{|c|}{$\begin{array}{c}\text { Post-pubertal } \\
\text { (21) }\end{array}$} \\
\hline & Mean & $\mathrm{SE}$ & Mean & $\mathrm{SE}$ & Mean & $\mathrm{SE}$ \\
\hline Age (years) & $11 \cdot 3$ & $0 \cdot 16$ & 13.8 & 0.21 & $17 \cdot 5$ & 0.39 \\
\hline Body-wt (kg) & $37 \cdot 3$ & 0.64 & $52 \cdot 2$ & $1 \cdot 45$ & $72 \cdot 3^{*}$ & $2 \cdot 33$ \\
\hline Body height $(\mathrm{m})$ & 1.478 & 0.0095 & $1 \cdot 661$ & 0.0015 & $1 \cdot 805^{*}$ & 0.0011 \\
\hline Body mass index $\left(\mathrm{kg} / \mathrm{m}^{2}\right)$ & $16 \cdot 9$ & $0 \cdot 15$ & $18 \cdot 8$ & $0 \cdot 35$ & $22 \cdot 1^{*}$ & $0 \cdot 61$ \\
\hline \multicolumn{7}{|l|}{ Skinfolds (mm) } \\
\hline Biceps & $5 \cdot 3$ & $0 \cdot 19$ & $5 \cdot 1$ & $0 \cdot 32$ & $5 \cdot 6$ & $0 \cdot 70$ \\
\hline Triceps & $9 \cdot 5$ & 0.31 & $9 \cdot 8$ & 0.56 & $10 \cdot 5$ & $1 \cdot 11$ \\
\hline Subscapular & $6 \cdot 2$ & 0.20 & $7 \cdot 2$ & 0.49 & $9 \cdot 7^{*}$ & 0.94 \\
\hline Supra-iliac & $7 \cdot 6$ & 0.42 & $8 \cdot 6$ & 0.88 & $14 \cdot 2^{*}$ & 1.92 \\
\hline Body density (kg/l) & $1 \cdot 0522$ & 0.0016 & $1 \cdot 0568$ & 0.0018 & $1 \cdot 0654^{*}$ & 0.0022 \\
\hline Body fat $(\%) \dagger$ & $15 \cdot 8$ & $0 \cdot 54$ & $15 \cdot 5$ & 0.74 & $13 \cdot 9$ & 0.98 \\
\hline Waist circumference $(\mathrm{mm})$ & 583 & 4.8 & 647 & $9 \cdot 1$ & $740^{*}$ & 13.5 \\
\hline Hip circumference (mm) & 741 & $4 \cdot 7$ & 823 & $10 \cdot 3$ & $932 *$ & $12 \cdot 0$ \\
\hline Waist : hip ratio & $0 \cdot 79$ & 0.0037 & 0.79 & 0.0085 & 0.79 & 0.0087 \\
\hline Trunk: total skinfolds & 0.48 & 0.005 & 0.51 & 00120 & $0.60^{*}$ & 0.0109 \\
\hline
\end{tabular}

* Mean values were significantly different from other age-groups, indicating an age trend $(P<0.05)$.

$\uparrow$ From body density.

\$ (Iliac + scapular): (biceps + triceps + iliac + scapular).

Table 2. Physical characteristics of prepubertal, pubertal and post-pubertal girls (Mean values with their standard errors; no. of girls in parentheses)

\begin{tabular}{|c|c|c|c|c|c|c|}
\hline & \multicolumn{2}{|c|}{$\begin{array}{c}\text { Prepubertal } \\
\text { (98) }\end{array}$} & \multicolumn{2}{|c|}{$\begin{array}{l}\text { Pubertal } \\
(76)\end{array}$} & \multicolumn{2}{|c|}{$\begin{array}{c}\text { Post-pubertal } \\
\text { (34) }\end{array}$} \\
\hline & Mean & SE & Mean & SE & Mean & SE \\
\hline Age (years) & $10 \cdot 5$ & $0 \cdot 16$ & $13 \cdot 1$ & 0.15 & 16.8 & $0-36$ \\
\hline Body-wt (kg) & $34 \cdot 0$ & 0.67 & $49 \cdot 8$ & 0.88 & $61.7^{*}$ & 1.47 \\
\hline Body height (m) & $1 \cdot 421$ & 0.0107 & $1 \cdot 621$ & 0.0073 & $1.697^{*}$ & 0.0115 \\
\hline Body mass index $\left(\mathrm{kg} / \mathrm{m}^{2}\right)$ & $16 \cdot 6$ & $0 \cdot 15$ & $18 \cdot 9$ & 0.25 & $21 \cdot 4^{*}$ & 0.41 \\
\hline \multicolumn{7}{|l|}{ Skinfolds (mm) } \\
\hline Biceps & $6 \cdot 6$ & $0 \cdot 25$ & $7 \cdot 6$ & 0.29 & $7 \cdot 7$ & 0.65 \\
\hline Triceps & $11 \cdot 9$ & $0 \cdot 42$ & $13 \cdot 5$ & $0 \cdot 50$ & 15.9 & 0.89 \\
\hline Subscapular & $7 \cdot 1$ & $0 \cdot 23$ & $8 \cdot 9$ & $0 \cdot 44$ & $10 \cdot 7^{*}$ & $0 \cdot 79$ \\
\hline Supra-iliac & $9 \cdot 7$ & 0.50 & $12 \cdot 0$ & 0.68 & $15 \cdot 0^{*}$ & $1 \cdot 20$ \\
\hline Body density $(\mathrm{kg} / \mathrm{l})$ & 1.0431 & 0.0013 & 1.0471 & 0.0110 & $1.0472^{*}$ & 0.0028 \\
\hline Body fat $(\%) \dagger$ & $19 \cdot 6$ & $0 \cdot 64$ & $19 \cdot 5$ & $0 \cdot 52$ & $21 \cdot 7$ & $1 \cdot 11$ \\
\hline Waist circumference (mm) & 566 & $5 \cdot 0$ & 633 & $6 \cdot 5$ & $683^{*}$ & $9 \cdot 4$ \\
\hline Hip circumference $(\mathrm{mm})$ & 736 & $5 \cdot 2$ & 845 & $7 \cdot 0$ & $925^{*}$ & 9.6 \\
\hline Waist: hip ratio & $0 \cdot 77$ & 0.0040 & 0.75 & 0.0057 & $0.74^{*}$ & 0.0086 \\
\hline Trunk : total skinfolds & $0 \cdot 47$ & 0.0050 & $0 \cdot 49$ & 0.0069 & $0.51^{*}$ & 0.0137 \\
\hline
\end{tabular}

* Mean values were significantly different from other age-groups, indicating an age trend $(P<0.05)$.

+ From body density.

$\ddagger$ (Iliac + scapular): (biceps + triceps + iliac + scapular). 
Table 3. Pearson's correlation coefficients for body density with log skinfolds for boys and girls of different maturation level

(No. of subjects in parentheses)

\begin{tabular}{|c|c|c|c|c|c|c|}
\hline Skinfold ... & Biceps & Triceps & Iliac & Scapular & Twot & Four $\ddagger$ \\
\hline \multicolumn{7}{|l|}{ Boys } \\
\hline Prepubertal (114) & $0.60^{* * *}$ & $0 \cdot 54 * * *$ & $0.52 * * *$ & $0 \cdot 59^{* * *}$ & $0.59 * * *$ & $0.61 * * *$ \\
\hline Pubertal (35) & $0.66^{* * *}$ & $0 \cdot 72^{* * *}$ & $0.25^{\mathrm{NS}}$ & $0.52 * * *$ & $0.73^{* * *}$ & $0 \cdot 60^{* * *}$ \\
\hline Post-pubertal (2l) & $0.69 * * *$ & $0 \cdot 72 * * *$ & $0 \cdot 72^{* * *}$ & $0 \cdot 69^{* * *}$ & $0.74 * * *$ & $0.76^{* * *}$ \\
\hline All $(170)$ & $0.58 * * *$ & $0.53 * * *$ & $0 \cdot 29 * * *$ & $0 \cdot 34^{* * *}$ & $0 \cdot 57^{* * *}$ & $0.56 * * *$ \\
\hline \multicolumn{7}{|l|}{ Girls } \\
\hline Prepubertal (98) & $0.61 * * *$ & $0.57 * * *$ & $0 \cdot 59 * * *$ & $0 \cdot 62 * * *$ & $0.61^{* * *}$ & $0 \cdot 64 * * *$ \\
\hline Pubertal (76) & $0 \cdot 70^{* * *}$ & $0.67 * * *$ & $0.53^{* * *}$ & $0 \cdot 64 * * *$ & $0.72 * * *$ & $0 \cdot 7 l^{* * *}$ \\
\hline Post-pubertal (34) & $0.71 * * *$ & $0.72 * * *$ & $0 \cdot 69^{* * *}$ & $0 \cdot 76^{* * *}$ & $0.75^{* * *}$ & $0.83^{* * *}$ \\
\hline All (208) & $0.59^{* * *}$ & $0.53 * * *$ & $0.49 * * *$ & $0 \cdot 52^{* * *}$ & $0.57^{* * *}$ & $0.58 * * *$ \\
\hline
\end{tabular}

NS, not significant.

*** $P<0.001$.

$\dagger \log$ (biceps + triceps).

$\neq \log$ (biceps + triceps + iliac + scapular).

Table 4. Prediction of body density from skinfold thicknesses and age for boys and girls of different maturation level

(Mean values and their standard errors)

\begin{tabular}{|c|c|c|c|c|c|c|c|c|c|c|}
\hline & \multicolumn{8}{|c|}{ Regression coefficients } & \multirow{3}{*}{\multicolumn{2}{|c|}{$\begin{array}{c}\text { SEE } \\
A(\mathrm{~kg} / 1 \\
\left.\left(\times 10^{-3}\right)\right)\end{array}$}} \\
\hline & \multicolumn{2}{|c|}{$\begin{array}{l}\text { Constant } \\
(\mathrm{kg} / \mathrm{l})\end{array}$} & \multicolumn{2}{|c|}{$\begin{array}{l}\text { Logtwo } \\
\text { skinfolds } \dagger\end{array}$} & \multicolumn{2}{|c|}{$\begin{array}{l}\text { Logfour } \\
\text { skinfolds } \ddagger\end{array}$} & \multicolumn{2}{|c|}{$\underset{\left(\text { years }\left(\times 10^{-3}\right)\right)}{\text { Age }}$} & & \\
\hline & Mean & $\mathrm{SE}$ & Mean & SE & Mean & $\mathrm{SE}$ & Mean & $\mathrm{SE}$ & & \\
\hline \multicolumn{11}{|l|}{ Boys } \\
\hline \multirow[t]{2}{*}{ Prepubertal } & $1.0963^{* * *}$ & 0.0091 & $-0.0564 * * *$ & 0.0066 & - & & $1 \cdot 8 * * *$ & 0.0005 & 0.41 & $9 \cdot 63$ \\
\hline & $1 \cdot 1133^{* * *}$ & 0.0010 & - & & $-0.0561 * * *$ & $0 \cdot 0063$ & $1.7^{*}$ & 0.0005 & 0.43 & 9.48 \\
\hline \multirow[t]{2}{*}{ Pubertal } & $1.0691^{* * *}$ & 0.0021 & $-0.0432^{* * *}$ & 0.00990 & - & & $2 \cdot 7 * *$ & 0.0001 & 0.61 & 6.68 \\
\hline & $1 \cdot 0555^{* * *}$ & 0.0022 & - & & $-0.0352 * * *$ & 0.0092 & $3 \cdot 8 * * *$ & 0.0001 & 0.55 & $7 \cdot 26$ \\
\hline \multirow[t]{2}{*}{ Post-pubertal } & $1 \cdot 1132^{* * *}$ & 0.0099 & $-0.0410^{* * *}$ & 0.0084 & - & & NS & - & 0.56 & 6.87 \\
\hline & $1 \cdot 1324 * * *$ & 0.0013 & - & & $-0.0429 * * *$ & 0.0083 & NS & - & 0.58 & $6 \cdot 64$ \\
\hline \multicolumn{11}{|l|}{ Girls } \\
\hline \multirow[t]{2}{*}{ Prepubertal } & $1 \cdot 0984^{* * *}$ & 0.0095 & $-0.0633^{* * *}$ & 0.0074 & - & & $2 \cdot 2 * * *$ & 0.0006 & 0.44 & $9 \cdot 80$ \\
\hline & $1 \cdot 1187^{* * *}$ & $0 \cdot 0011$ & - & & $-0 \cdot 0630^{* * *}$ & 0.0069 & $1.9 * *$ & 0.0006 & 0.47 & 9.54 \\
\hline \multirow{2}{*}{ Pubertal } & $1 \cdot 1003^{* * *}$ & 0.0011 & $-0.0552 * * *$ & 0.0059 & - & & $1 \cdot 4^{*}$ & $0 \cdot 0058$ & 0.56 & $6 \cdot 50$ \\
\hline & $1 \cdot 1074^{* * *}$ & 0.0012 & - & & $-0 \cdot 0504 * * *$ & 0.0056 & $1 \cdot 6^{* *}$ & $0 \cdot 0059$ & 0.54 & $6 \cdot 60$ \\
\hline \multirow[t]{2}{*}{ Post-pubertal } & $1 \cdot 1547 * * *$ & 0.0017 & $-0.0796^{* * *}$ & 0.0012 & - & & NS & - & 0.56 & $9 \cdot 33$ \\
\hline & $1 \cdot 1830^{* * *}$ & 0.0016 & - & & $-0.0813^{* * *}$ & 0.0097 & NS & - & 0.69 & $7 \cdot 86$ \\
\hline
\end{tabular}

$A$, percentage of explained variance.

SEE, standard error of estimate; NS, not significant

$* P<0.05$, ** $P<0.01$, *** $P<0.001$.

$\dagger \log ($ biceps + triceps $)$.

$\ddagger \log ($ biceps + triceps + iliac + scapular).

\section{RESULTS}

Tables 1 and 2 show some of the physical characteristics of the male and female study population, divided into three levels of maturation status. In general, at each maturation level boys were older, had higher body-weights and body heights, thinner skinfolds, higher 
Table 5. Prediction of body fat percentage from skinfold thicknesses in boys and girls of different maturation level

(Mean values with their standard errors)

\begin{tabular}{|c|c|c|c|c|c|c|c|c|}
\hline & \multicolumn{6}{|c|}{ Regression coefficients } & \multirow[b]{3}{*}{$A$} & \multirow{3}{*}{$\begin{array}{l}\text { SEE } \\
(\%)\end{array}$} \\
\hline & \multicolumn{2}{|c|}{$\begin{array}{c}\text { Constant } \\
(\%)\end{array}$} & \multicolumn{2}{|c|}{$\begin{array}{l}\text { Logtwo } \\
\text { skinfolds } \dagger\end{array}$} & \multicolumn{2}{|c|}{$\begin{array}{l}\text { Logfour } \\
\text { skinfolds } \ddagger\end{array}$} & & \\
\hline & Mean & $\mathrm{SE}$ & Mean & $\mathrm{SE}$ & Mean & $\mathrm{SE}$ & & \\
\hline \multicolumn{9}{|l|}{ Boys } \\
\hline Prepubertal & $\begin{array}{l}-14 \cdot 61^{* * *} \\
-22 \cdot 23^{* * *}\end{array}$ & $\begin{array}{l}3 \cdot 61 \\
4 \cdot 31\end{array}$ & $26 \cdot 51^{* * *}$ & $3 \cdot 13$ & $26 \cdot 56^{* * *}$ & 3.00 & $\begin{array}{l}0.39 \\
0.41\end{array}$ & $\begin{array}{l}5 \cdot 6 \\
4 \cdot 5\end{array}$ \\
\hline \multirow[t]{2}{*}{ Pubertal } & $-9 \cdot 78^{*}$ & $4 \cdot 35$ & $21 \cdot 90^{* * *}$ & 3.75 & 2000 & 500 & 0.51 & $3 \cdot 1$ \\
\hline & $-11.91^{*}$ & $6 \cdot 34$ & $1777 * *$ & & $18 \cdot 70^{* * *}$ & $4 \cdot 31$ & 0.36 & $3 \cdot 5$ \\
\hline Post-pubertal & $\begin{array}{c}-6 \cdot 81^{\mathrm{NS}} \\
-15 \cdot 58^{*}\end{array}$ & $\begin{array}{l}4 \cdot 61 \\
5 \cdot 96\end{array}$ & $17.77^{* * *}$ & 3.91 & $18 \cdot 88^{* * *}$ & 3.79 & $\begin{array}{l}0.52 \\
0.57\end{array}$ & $\begin{array}{l}3 \cdot 2 \\
3 \cdot 0\end{array}$ \\
\hline \multicolumn{9}{|l|}{ Girls } \\
\hline \multirow[t]{2}{*}{ Prepubertal } & $\begin{array}{l}-16 \cdot 84^{* * *} \\
-25 \cdot 87^{* * *}\end{array}$ & $\begin{array}{l}4 \cdot 26 \\
4.96\end{array}$ & $29 \cdot 30^{* * *}$ & $3 \cdot 40$ & - & & 0.44 & $4 \cdot 8$ \\
\hline & $\begin{array}{l}-25 \cdot 87^{* * *} \\
-14 \cdot 79^{* * *}\end{array}$ & $\begin{array}{l}4.96 \\
3.65\end{array}$ & $26 \cdot 21 * * *$ & & $29 \cdot 85^{* * *}$ & $3 \cdot 25$ & 0.47 & $4 \cdot 7$ \\
\hline Pubertal & $-18.89^{* * *}$ & $\begin{array}{l}5.05 \\
4.20\end{array}$ & 20.21 & $2 \cdot 78$ & $23 \cdot 94 * * *$ & 2.61 & 0.54 & $3 \cdot 0$ \\
\hline \multirow[t]{2}{*}{ Post-pubertal } & $-27.71^{* *}$ & $8 \cdot 16$ & $36.53^{* * *}$ & $6 \cdot 01$ & & & 0.54 & $4 \cdot 5$ \\
\hline & $-43 \cdot 49 * * *$ & 7.25 & . & & $39 \cdot 02^{* * *}$ & $4 \cdot 33$ & 0.71 & $3 \cdot 5$ \\
\hline
\end{tabular}

$A$, percentage of explained variance.

SEE, standard error of estimate; NS, not significant.

* $P<0.05, * * P<0.01, * * * P<0.001$.

$\dagger \log$ (biceps + triceps).

$\$ \log$ (biceps + triceps + iliac + scapular $)$.

Table 6. Pearson's correlation coefficients of waist: hip ratio with trunk:total skinfold thickness ratio $\dagger$ for boys and girls of different maturation level

\begin{tabular}{|c|c|c|c|}
\hline & Prepubertal & Pubertal & Post-pubertal \\
\hline Boys & $0 \cdot 16$ & $0 \cdot 19$ & $0.41^{*}$ \\
\hline Girls & $0 \cdot 11$ & $0.22^{*}$ & $0.45^{* *}$ \\
\hline
\end{tabular}

* $P<0.05,{ }^{* *} P<0.01$.

$\dagger$ (Iliac + scapular): (biceps + triceps + iliac + scapular).

body densities, lower percentage body fat, higher waist:hips ratios and higher trunk : total skinfold ratios compared with girls.

Table 3 gives the Pearson's correlation coefficients for body density with the log of the separate skinfolds, the log of the sum of biceps and triceps (logtwo) and the log of the sum of all four skinfolds (logfour) for boys and girls in the separate maturation groups. In general the correlation coefficients were slightly higher in the older age groups. Because in most subgroups the correlation of body density with logtwo and logfour was as good as or better than the correlation of body density with the separate skinfolds, further analyses were performed with logtwo and logfour only as independent variables.

In Table 4 the (multiple) regression equations with explained variance $(A)$ and standard error of estimate (SEE) of body density as the dependent variable and logtwo (or logfour) and age as independent variables are given for boys and girls in the maturation subgroups. 
Table 7. Percentage body fat estimated from the sum of biceps and triceps skinfold in boys of different maturation level

(Mean values and $95 \%$ confidence interval, CI)

\begin{tabular}{|c|c|c|c|c|c|c|}
\hline \multirow{3}{*}{$\begin{array}{l}\text { Sum of } \\
\text { skinfolds }\end{array}$} & \multicolumn{6}{|c|}{ Percentage body fat } \\
\hline & \multicolumn{2}{|c|}{$\begin{array}{c}\text { Prepubertal } \\
11 \cdot 3 \text { (SE 1.7)years }\end{array}$} & \multicolumn{2}{|c|}{$\begin{array}{c}\text { Pubertal } \\
13.8 \text { (SE 1.3)years }\end{array}$} & \multicolumn{2}{|c|}{$\begin{array}{c}\text { Post-pubertal } \\
17.5(\text { SE I.8) years }\end{array}$} \\
\hline & Mean & $95 \% \mathrm{CI}$ & Mean & $95 \% \mathrm{CI}$ & Mean & $95 \% \mathrm{CI}$ \\
\hline 6 & $6 \cdot 1$ & $5 \cdot 1-7 \cdot 1$ & $7 \cdot 3$ & $6 \cdot 3-8 \cdot 3$ & $7 \cdot 0$ & $5 \cdot 5-8 \cdot 5$ \\
\hline 7 & $7 \cdot 8$ & $6.8-8.8$ & $8 \cdot 7$ & $7 \cdot 7-9 \cdot 7$ & $8 \cdot 2$ & $6 \cdot 7-9 \cdot 7$ \\
\hline 8 & $9 \cdot 0$ & $8 \cdot 0-10 \cdot 0$ & $10 \cdot 0$ & $9 \cdot 0-11 \cdot 0$ & $9 \cdot 2$ & $7 \cdot 7-10 \cdot 7$ \\
\hline 9 & 10.7 & $9 \cdot 7-11.7$ & $11 \cdot 1$ & $10 \cdot 1-12 \cdot 1$ & $10 \cdot 1$ & $8 \cdot 6-11.6$ \\
\hline 10 & $12 \cdot 0$ & $11 \cdot 0-130$ & $12 \cdot 1$ & $11 \cdot 1-13 \cdot 1$ & $11 \cdot 0$ & $9 \cdot 5-12 \cdot 5$ \\
\hline 11 & 13.0 & $12 \cdot 0-14 \cdot 0$ & $13 \cdot 0$ & $12 \cdot 0-14 \cdot 0$ & $11 \cdot 7$ & $10 \cdot 2-13 \cdot 2$ \\
\hline 12 & $14 \cdot 1$ & $13 \cdot 1-15 \cdot 1$ & $13 \cdot 9$ & $12 \cdot 9-14 \cdot 9$ & 12.4 & $10 \cdot 9-13 \cdot 9$ \\
\hline 13 & 150 & $14 \cdot 0-16.0$ & 14.6 & $13 \cdot 6-15 \cdot 6$ & $13 \cdot 0$ & $11 \cdot 5-14.5$ \\
\hline 14 & $15 \cdot 8$ & $14 \cdot 8-16 \cdot 8$ & $15 \cdot 3$ & $14 \cdot 3-16 \cdot 3$ & 13.6 & $12 \cdot 1-15 \cdot 1$ \\
\hline 15 & 166 & $15.6-17.6$ & 16.0 & $15 \cdot 0-17 \cdot 0$ & $14 \cdot 1$ & $12 \cdot 6-15 \cdot 6$ \\
\hline 16 & $17 \cdot 4$ & $16 \cdot 4-18.4$ & $16 \cdot 6$ & $15 \cdot 6-17 \cdot 6$ & 14.6 & $13 \cdot 1-16 \cdot 1$ \\
\hline 17 & $18 \cdot 1$ & $17 \cdot 4-19 \cdot 1$ & $17 \cdot 2$ & $16 \cdot 2-18 \cdot 2$ & $15 \cdot 1$ & $13 \cdot 6-16 \cdot 6$ \\
\hline 18 & 18.7 & $17 \cdot 7-19 \cdot 7$ & $17 \cdot 7$ & $16 \cdot 7-18 \cdot 7$ & $15 \cdot 5$ & $14.0-17.0$ \\
\hline 19 & $19 \cdot 4$ & $18 \cdot 4-20 \cdot 4$ & $18 \cdot 2$ & $17 \cdot 2-19 \cdot 2$ & 15.9 & $14 \cdot 4-17 \cdot 4$ \\
\hline 20 & 19.9 & $18 \cdot 9-20 \cdot 9$ & 187 & $17 \cdot 7-19 \cdot 7$ & $16 \cdot 3$ & $14 \cdot 8-17 \cdot 8$ \\
\hline 22 & $21 \cdot 0$ & $20 \cdot 0-22 \cdot 0$ & $19 \cdot 6$ & $18 \cdot 6-20 \cdot 6$ & $17 \cdot 0$ & $15 \cdot 5-18.5$ \\
\hline 24 & $22 \cdot 0$ & $21 \cdot 0-23 \cdot 0$ & $20 \cdot 4$ & $19 \cdot 4-21 \cdot 4$ & 17.7 & $16 \cdot 2-19 \cdot 2$ \\
\hline 26 & 23.0 & $22 \cdot 0-24 \cdot 0$ & $21 \cdot 2$ & $20 \cdot 1-22 \cdot 1$ & $18 \cdot 3$ & $16 \cdot 8-19 \cdot 8$ \\
\hline 28 & 23.8 & $22 \cdot 8-24 \cdot 8$ & $21 \cdot 9$ & $20 \cdot 9-22 \cdot 9$ & 18.9 & $17 \cdot 4-20 \cdot 4$ \\
\hline 30 & $24 \cdot 6$ & $23.6-25.6$ & $22 \cdot 6$ & $21 \cdot 6-23 \cdot 6$ & $19 \cdot 4$ & $17 \cdot 9-20 \cdot 9$ \\
\hline 35 & $26 \cdot 4$ & $25 \cdot 4-27 \cdot 4$ & $24 \cdot 0$ & $23 \cdot 0-35 \cdot 0$ & $20 \cdot 6$ & $19 \cdot 1--22 \cdot 1$ \\
\hline 40 & 27.9 & $26 \cdot 9-28 \cdot 9$ & $25 \cdot 3$ & $24 \cdot 3-26 \cdot 3$ & $21 \cdot 7$ & $20 \cdot 2-23 \cdot 2$ \\
\hline
\end{tabular}

As can be seen, age is still an important variable in the prediction of body density in both prepubertal and in pubertal boys and girls, but not in post-pubertal boys and girls.

In Table 5 the prediction equations for percentage body fat from skinfolds (logtwo or logfour) are given for boys and girls of different maturation levels. In these prediction formulas age did not contribute significantly in either subgroup. In the appendix (Tables 7-10) the percentage body fat and the $95 \%$ confidence interval for boys and girls of different maturation level are given, estimated from the sum of two or the sum of four skinfolds.

In all maturity groups the waist: hips ratio in boys was significantly higher than in girls, mainly due to a higher waist circumference in boys. In girls, but not in boys, the waist : hips ratio decreased with age (Tables 1 and 2).

Only in the older boys and girls did the waist: hips ratio correlate with measures of body fatness. In post-pubertal boys the waist:hips ratio correlated with logfour $(r 0.58$, $P<0.01)$, logbiceps $(r 0.55, P<0.01)$, logsupra-iliac $(r 0.63, P<0.01)$ and logsubscapular $(r 0.58, P<0.01)$. In pubertal and post-pubertal girls the waist:hips ratio was correlated with logsupra-iliac only $(r 0.30, P<0.01$ and $r 0.41, P<0.01$ respectively).

In post-pubertal boys and in pubertal and post-pubertal girls the waist:hips ratio was correlated with another measure of body fat distribution (trunk skinfolds : total skinfolds). The relationship between the waist:hips ratio and the skinfold ratio at the different maturation levels in boys and girls is shown in Table 6 . 
Table 8. Percentage body fat estimated from the sum of biceps and triceps skinfold in girls of different maturation level

(Mean values and $95 \%$ confidence interval, $\mathrm{CI}$ )

\begin{tabular}{|c|c|c|c|c|c|c|}
\hline \multirow{3}{*}{$\begin{array}{l}\text { Sum of } \\
\text { skinfolds }\end{array}$} & \multicolumn{6}{|c|}{ Percentage body fat } \\
\hline & \multicolumn{2}{|c|}{$\begin{array}{l}\text { Prepubertal } \\
11 \cdot 7 \text { (SE } 1 \cdot 7 \text { ) years }\end{array}$} & \multicolumn{2}{|c|}{$\begin{array}{l}\text { Pubertal } \\
13 \cdot 8 \text { (SE 1.3) years }\end{array}$} & \multicolumn{2}{|c|}{$\begin{array}{c}\text { Post-pubertal } \\
17.5(\mathrm{SE} 1.8) \text { years }\end{array}$} \\
\hline & Mean & $95 \% \mathrm{CI}$ & Mean & $95 \%$ CI & Mean & $95 \% \mathrm{CI}$ \\
\hline 8 & $9 \cdot 6$ & $8 \cdot 6-10 \cdot 6$ & $8 \cdot 9$ & $7 \cdot 9-9 \cdot 9$ & - & - . \\
\hline 9 & $11 \cdot 1$ & $10 \cdot 1-12 \cdot 1$ & $10 \cdot 2$ & $9 \cdot 2-11 \cdot 2$ & - & - \\
\hline 10 & $12 \cdot 5$ & $11 \cdot 5-13 \cdot 5$ & 11.4 & $10 \cdot 4-12 \cdot 4$ & $8 \cdot 8$ & $7 \cdot 3-10 \cdot 3$ \\
\hline 11 & $13 \cdot 7$ & $12 \cdot 7-14 \cdot 7$ & $12 \cdot 5$ & $11 \cdot 5-13 \cdot 5$ & $10 \cdot 3$ & $8 \cdot 8-11 \cdot 8$ \\
\hline 12 & $14 \cdot 8$ & $13 \cdot 8-15 \cdot 8$ & $13 \cdot 5$ & $12 \cdot 5-14.5$ & $11 \cdot 7$ & $10 \cdot 2-13 \cdot 2$ \\
\hline 13 & $15 \cdot 8$ & $14 \cdot 8-16 \cdot 8$ & $14 \cdot 4$ & $13 \cdot 4-15 \cdot 4$ & $13 \cdot 0$ & $11 \cdot 5-14 \cdot 5$ \\
\hline 14 & 167 & $15 \cdot 7-17 \cdot 7$ & $15 \cdot 3$ & $14 \cdot 3-16 \cdot 3$ & $14 \cdot 2$ & $12 \cdot 7-15 \cdot 7$ \\
\hline 15 & $17 \cdot 6$ & $16 \cdot 7 \ldots 18 \cdot 7$ & 160 & $15 \cdot 0-17 \cdot 0$ & $15 \cdot 3$ & $13 \cdot 8-16 \cdot 8$ \\
\hline 16 & $18 \cdot 4$ & $17 \cdot 4-19 \cdot 4$ & 168 & $15 \cdot 8-17 \cdot 8$ & $16 \cdot 3$ & $14.8-17 \cdot 8$ \\
\hline 17 & $19 \cdot 2$ & $18 \cdot 2-20 \cdot 2$ & $17 \cdot 5$ & $16 \cdot 5-18 \cdot 5$ & $17 \cdot 2$ & $15 \cdot 7-18 \cdot 7$ \\
\hline 18 & $19 \cdot 9$ & $18.9-20 \cdot 9$ & $18 \cdot 1$ & $17 \cdot 1-19 \cdot 1$ & $18 \cdot 1$ & $16 \cdot 6-19 \cdot 6$ \\
\hline 19 & $20 \cdot 6$ & $19 \cdot 6-21 \cdot 6$ & 18.7 & $17 \cdot 7-19 \cdot 7$ & $19 \cdot 0$ & $17 \cdot 5-20 \cdot 5$ \\
\hline 20 & $21 \cdot 3$ & $20 \cdot 3-22 \cdot 3$ & $19 \cdot 3$ & $18 \cdot 3-20 \cdot 3$ & $19 \cdot 8$ & $18 \cdot 3-21 \cdot 3$ \\
\hline 22 & $22 \cdot 5$ & $21 \cdot 5-23 \cdot 5$ & $20 \cdot 4$ & $19 \cdot 4-21 \cdot 4$ & $21 \cdot 3$ & $19 \cdot 8-22 \cdot 8$ \\
\hline 24 & $23 \cdot 6$ & $20 \cdot 6-22 \cdot 6$ & $21 \cdot 4$ & $20.4-22.4$ & $22 \cdot 7$ & $21 \cdot 2-24 \cdot 2$ \\
\hline 26 & $24 \cdot 6$ & $23 \cdot 6-25 \cdot 6$ & $22 \cdot 3$ & $21 \cdot 3-23 \cdot 3$ & $24 \cdot 0$ & $22 \cdot 5-25 \cdot 5$ \\
\hline 28 & $25 \cdot 6$ & $24 \cdot 6-26 \cdot 6$ & $23 \cdot 1$ & $22 \cdot 1-24 \cdot 1$ & $25 \cdot 2$ & $23 \cdot 7-26 \cdot 7$ \\
\hline 30 & $26 \cdot 4$ & $25 \cdot 4-27 \cdot 4$ & $23 \cdot 9$ & $22 \cdot 9-24 \cdot 9$ & $26 \cdot 2$ & $24 \cdot 7-27 \cdot 7$ \\
\hline 35 & $28 \cdot 4$ & $27 \cdot 4-29 \cdot 4$ & $25 \cdot 7$ & $24 \cdot 7-26 \cdot 7$ & $28 \cdot 7$ & $27 \cdot 2-30 \cdot 2$ \\
\hline 40 & $30 \cdot 1$ & $29 \cdot 1-31 \cdot 1$ & $27 \cdot 2$ & $26 \cdot 2-28 \cdot 2$ & 30.8 & $29 \cdot 3-32 \cdot 3$ \\
\hline 45 & $31 \cdot 6$ & $30 \cdot 6-32 \cdot 6$ & 28.5 & $27 \cdot 5-29 \cdot 5$ & $32 \cdot 7$ & $30 \cdot 2-33 \cdot 2$ \\
\hline 50 & $32 \cdot 9$ & $31 \cdot 9-33.9$ & $29 \cdot 7$ & $28 \cdot 7-30 \cdot 7$ & $34 \cdot 4$ & $32 \cdot 9-35 \cdot 9$ \\
\hline
\end{tabular}

\section{DISCUSSION}

The study population was recruited from some arbitrarily chosen primary and secondary schools. For the purpose of this methodological study a representative sample was not required. However, when the body-weights and body heights of the studied population are compared with values for a representative sample of the Dutch population (Roede \& van Wieringen, 1985), no striking differences are observed. The anthropometric variables are also in good agreement with the findings of a recently published study of 2285 Dutch children aged 0-18 years (Weststrate et al. 1989).

Body densities in boys were in general higher than in girls, the differences being smallest in the younger age-groups. A similar finding was made in an earlier study (Deurenberg et al. 1989 b) and also recently by Slaughter et al. (1988). The body density values found in the present study are in good agreement with the predicted values derived from theoretical equations published by Weststrate \& Deurenberg (1989), which used the same equations for boys and girls, i.e. the same density for the fat-free mass in boys and girls at a given age. The mean (with SE) difference (measured-predicted density) in boys was -0.0006 (SE 0.0007) kg/l and in girls -0.0013 (SE 0.0006) kg/l, corresponding to a difference in calculated percentage body fat of less than $1 \%$, a difference which is biologically meaningless. When using the prediction equation for girls as published in the original model, the mean (with $\mathrm{SE}$ ) difference between the measured and the predicted density would 
Table 9. Percentage body fat estimated from the sum of biceps, triceps, supra-iliac and subscapular skinfold in boys of different maturation level

(Mean value and $95 \%$ confidence interval, $\mathrm{CI}$ )

\begin{tabular}{|c|c|c|c|c|c|c|}
\hline \multirow{3}{*}{$\begin{array}{l}\text { Sum of } \\
\text { skinfolds }\end{array}$} & \multicolumn{6}{|c|}{ Percentage body fat } \\
\hline & \multicolumn{2}{|c|}{$\begin{array}{c}\text { Prepubertal } \\
10.5 \text { (SE 1.6) years }\end{array}$} & \multicolumn{2}{|c|}{$\begin{array}{c}\text { Pubertal } \\
13 \cdot 2 \text { (SE 1-3)years }\end{array}$} & \multicolumn{2}{|c|}{$\begin{array}{c}\text { Post-pubertal } \\
16.8(\mathrm{SE} 2 \cdot 1) \text { years }\end{array}$} \\
\hline & Mean & $95 \% \mathrm{CI}$ & Mean & $95 \% \mathrm{CI}$ & Mean & $95 \% \mathrm{CI}$ \\
\hline 15 & $9 \cdot 0$ & $8 \cdot 2-9 \cdot 8$ & $10 \cdot 1$ & $8 \cdot 9-11 \cdot 3$ & - & $\ldots$ \\
\hline 20 & $12 \cdot 3$ & $11 \cdot 5-13 \cdot 1$ & $12 \cdot 4$ & $11.2-13.6$ & $8-7$ & $7 \cdot 3-10 \cdot 1$ \\
\hline 25 & 14.9 & $14 \cdot 1-15 \cdot 7$ & $14 \cdot 2$ & $13 \cdot 0-15 \cdot 4$ & $10-5$ & $9 \cdot 1-11.9$ \\
\hline 30 & $17 \cdot 0$ & $16 \cdot 2-17 \cdot 8$ & 15.7 & $14 \cdot 5-16 \cdot 9$ & $12 \cdot 0$ & $10 \cdot 6-13 \cdot 4$ \\
\hline 35 & $18 \cdot 8$ & $18 \cdot 0-19 \cdot 6$ & $17 \cdot 0$ & $15 \cdot 8-18 \cdot 2$ & $13 \cdot 3$ & $11 \cdot 9-14 \cdot 7$ \\
\hline 40 & $20 \cdot 3$ & $19 \cdot 5-21 \cdot 1$ & 18.0 & $16 \cdot 8-19 \cdot 2$ & $14 \cdot 4$ & $13 \cdot 0-15 \cdot 8$ \\
\hline 45 & $21 \cdot 7$ & $20 \cdot 9-22 \cdot 5$ & $19 \cdot 0$ & $17 \cdot 8-20 \cdot 2$ & $15 \cdot 4$ & $14 \cdot 0-16.8$ \\
\hline 50 & $22 \cdot 9$ & $22 \cdot 1-23 \cdot 7$ & $19 \cdot 9$ & $18 \cdot 7-21 \cdot 2$ & $16 \cdot 2$ & $14.8-17.6$ \\
\hline 55 & $24 \cdot 0$ & $23 \cdot 2-24 \cdot 8$ & $20 \cdot 6$ & $19 \cdot 4-21 \cdot 8$ & $17 \cdot 0$ & $15 \cdot 6-18 \cdot 4$ \\
\hline 60 & $25 \cdot 0$ & $24 \cdot 2-25 \cdot 8$ & $21 \cdot 3$ & $20 \cdot 1-22 \cdot 5$ & 17.7 & $16 \cdot 3-19 \cdot 1$ \\
\hline 65 & $25 \cdot 9$ & $25 \cdot 1-26 \cdot 7$ & $22 \cdot 0$ & $20 \cdot 8-23 \cdot 2$ & $18 \cdot 4$ & $17 \cdot 0-19 \cdot 8$ \\
\hline 70 & $26 \cdot 8$ & $26 \cdot 0-27 \cdot 6$ & $22 \cdot 6$ & $21.4-23.8$ & $19 \cdot 0$ & $17 \cdot 6-20 \cdot 4$ \\
\hline 75 & $27 \cdot 6$ & $26 \cdot 8-28 \cdot 4$ & $23 \cdot 2$ & $22 \cdot 0-24 \cdot 4$ & 19.6 & $18 \cdot 2-21 \cdot 0$ \\
\hline 80 & $28 \cdot 3$ & $27 \cdot 5-29 \cdot 1$ & 23.7 & $22 \cdot 5-25 \cdot 9$ & $20-1$ & $18 \cdot 7-21.5$ \\
\hline 85 & $29 \cdot 0$ & $28 \cdot 2-29 \cdot 8$ & $24 \cdot 7$ & $23 \cdot 5-25 \cdot 9$ & $20 \cdot 6$ & $19 \cdot 2-22 \cdot 0$ \\
\hline 90 & $29 \cdot 7$ & $29 \cdot 1-30 \cdot 5$ & $24 \cdot 6$ & $24 \cdot 4-25 \cdot 8$ & $21 \cdot 0$ & $19 \cdot 6-22 \cdot 4$ \\
\hline 95 & $30 \cdot 3$ & $29 \cdot 5-31 \cdot 1$ & $25 \cdot 1$ & $23 \cdot 9-26 \cdot 3$ & $21 \cdot 5$ & $20 \cdot 1-22 \cdot 9$ \\
\hline
\end{tabular}

Table 10. Percentage body fat estimated from the sum of biceps, triceps, supra-iliac and subscapular skinfold in girls of different maturation level

(Mean value and $95 \%$ confidence interval, $\mathrm{CI}$ )

\begin{tabular}{|c|c|c|c|c|c|c|}
\hline \multirow{3}{*}{$\begin{array}{l}\text { Sum of } \\
\text { skinfolds }\end{array}$} & \multicolumn{6}{|c|}{ Percentage body fat } \\
\hline & \multicolumn{2}{|c|}{$\begin{array}{c}\text { Prepubertal } \\
10.5 \text { (SE } 1.6) \text { years }\end{array}$} & \multicolumn{2}{|c|}{$\begin{array}{l}\text { Pubertal } \\
13 \cdot 1 \text { (SE 1-3) years }\end{array}$} & \multicolumn{2}{|c|}{$\begin{array}{c}\text { Post-pubertal } \\
16-8 \text { (SE } 2 \cdot 1 \text { ) years }\end{array}$} \\
\hline & Mean & $95 \% \mathrm{CI}$ & Mean & $95 \% \mathrm{CI}$ & Mean & $95 \% \mathrm{CI}$ \\
\hline 15 & $9 \cdot 2$ & $8 \cdot 3-10 \cdot 1$ & $9 \cdot 3$ & $8 \cdot 6-10 \cdot 0$ & - & - \\
\hline 20 & $13 \cdot 0$ & $12 \cdot 1-13 \cdot 9$ & $12 \cdot 3$ & $9 \cdot 6-13 \cdot 0$ & - & - \\
\hline 25 & 15.9 & $15 \cdot 0-16 \cdot 8$ & $14 \cdot 6$ & $13 \cdot 9-15 \cdot 3$ & $11 \cdot 1$ & $9 \cdot 9-12 \cdot 3$ \\
\hline 30 & $18 \cdot 2$ & $17 \cdot 3-19 \cdot 1$ & $16 \cdot 5$ & $15 \cdot 8-17 \cdot 2$ & $14 \cdot 1$ & $12 \cdot 9-15 \cdot 3$ \\
\hline 35 & $20 \cdot 2$ & $19 \cdot 3-21 \cdot 1$ & $18 \cdot 1$ & $17 \cdot 4-18 \cdot 8$ & 168 & $15 \cdot 6-18 \cdot 0$ \\
\hline 40 & $22 \cdot 0$ & $21 \cdot 1-22 \cdot 9$ & $19 \cdot 5$ & $18 \cdot 8-20 \cdot 2$ & $19 \cdot 0$ & $17 \cdot 8-20 \cdot 2$ \\
\hline 45 & $23 \cdot 5$ & $22 \cdot 6-24 \cdot 4$ & $20 \cdot 7$ & $20 \cdot 0-21 \cdot 4$ & $21 \cdot 0$ & $19 \cdot 8-22 \cdot 2$ \\
\hline 50 & $24 \cdot 8$ & $23 \cdot 9-25 \cdot 7$ & $21 \cdot 8$ & $21 \cdot 1-22 \cdot 5$ & $22 \cdot 8$ & $21 \cdot 6-24 \cdot 0$ \\
\hline 55 & $26 \cdot 1$ & $25 \cdot 2-27 \cdot 0$ & $22 \cdot 8$ & $22 \cdot 1-23 \cdot 5$ & $24 \cdot 4$ & $23 \cdot 2-25 \cdot 6$ \\
\hline 60 & $27 \cdot 2$ & $26 \cdot 3-28 \cdot 1$ & $23 \cdot 7$ & $23 \cdot 0-24 \cdot 4$ & $25-9$ & $24 \cdot 7-27 \cdot 1$ \\
\hline 65 & $28 \cdot 2$ & $27 \cdot 3-29 \cdot 1$ & $24 \cdot 5$ & $23 \cdot 8-25 \cdot 2$ & $27 \cdot 2$ & $26 \cdot 0-28 \cdot 4$ \\
\hline 70 & $29 \cdot 2$ & $28 \cdot 3-30 \cdot 1$ & $25 \cdot 3$ & $24 \cdot 6-26 \cdot 0$ & $28 \cdot 5$ & $27 \cdot 3-29 \cdot 7$ \\
\hline 75 & $30 \cdot 1$ & $29 \cdot 2-31 \cdot 0$ & $26 \cdot 0$ & $25 \cdot 3-26 \cdot 7$ & $29-7$ & $28 \cdot 5-30 \cdot 9$ \\
\hline 80 & $30 \cdot 9$ & $30 \cdot 0-31 \cdot 8$ & $26 \cdot 7$ & $26 \cdot 0-27 \cdot 4$ & $30 \cdot 8$ & $29 \cdot 6-32 \cdot 0$ \\
\hline 85 & $31 \cdot 7$ & $30 \cdot 8-32 \cdot 6$ & $27 \cdot 3$ & $26 \cdot 6-28 \cdot 0$ & $31 \cdot 8$ & $30 \cdot 6-33 \cdot 0$ \\
\hline 90 & $32 \cdot 5$ & $31 \cdot 6-33 \cdot 4$ & 27.9 & $27 \cdot 2-28 \cdot 6$ & 32.8 & $31 \cdot 6-34 \cdot 0$ \\
\hline 95 & $33 \cdot 2$ & $32 \cdot 3-34 \cdot 1$ & 28.5 & $27 \cdot 8-29 \cdot 2$ & $33 \cdot 7$ & $32 \cdot 5-34 \cdot 9$ \\
\hline
\end{tabular}


be -0.0111 (SE 0.0006$) \mathrm{kg} / \mathrm{l}$ (corresponding to about $5 \%$ body fat), an error which is about ten times larger.

Skinfold thicknesses correlated well with body density in the three maturation age groups, with the exception of the very low correlation of the supra-iliac skinfold in pubertal boys (Table 3 ). In general the correlation coefficients were higher in the older age groups. This can be explained by the fact that the accuracy of the determination of body density in younger children is less than that in older children and adults (Mendez \& Lukaski, 1981; Deurenberg et al. 1989 b). Furthermore, the compressibility of the skinfolds is higher in younger children (Durnin \& Womersley, 1974), due to the higher water content in the adipose tissue, which will result in larger errors in the measurements and consequently in lower correlations with body density.

The correlation coefficient of the sum of two skinfolds (biceps + triceps) with body density was in both boys and girls comparable to the correlation of the sum of four skinfolds with body density. The prediction of the body density from the sum of two skinfolds or the sum of four skinfolds was still age-dependent in prepubertal and pubertal boys and girls, but not in post-pubertal boys and girls. This age dependency shows that the classification into three groups of maturation level is in fact a very approximate one. In a multiple regression model for the prediction of body density when, in addition to skinfolds, age (years) and maturation level are included as independent variables, age has always been selected first in the model. When maturation level was included in the model, the decrease in SEE was not marked. The error in the prediction of the body density from skinfolds in this young population (SEE 0.0065-0.0098 kg/l) was comparable to the error found by other authors in several populations (Durnin \& Womersley, 1974; Slaughter et al. 1988).

The prediction of the percentage body fat from skinfold thicknesses for both boys and girls of each maturation level was not age-dependent. This means that in the prediction formula of percentage body fat from body density as given previously (p. 294), the agedependent increase in the density of the fat-free mass is compensated.

As is shown in Table 5, the prediction error in deriving the percentage body fat from four skinfolds is only slightly lower than that from only two skinfolds. This observation, which has also been reported by other workers (Durnin \& Womersley, 1974) and which has also been found in our laboratory in adult subjects ( $P$. Deurenberg, unpublished results) and in elderly subjects (Deurenberg et al. 1989a), can be of practical importance. It is often difficult to measure four skinfolds or to measure trunk skinfolds in field studies, due to lack of time or to lack of cooperation of the subjects (unwillingness to undress).

Prediction formulas for percentage body fat from triceps and subscapula skinfolds, as recently published by Slaughter et al. (1988), give accurate estimates for percentage body fat from densitometric determinations. The differences (estimated minus predicted percentage body fat) were in prepubertal, pubertal and post-pubertal children respectively 0.7 (not significant, NS), 0.8 (NS) and -1.4 (NS) in boys and $2.3(P<0.001),-0.2(\mathrm{NS})$ and -0.4 (NS) in girls. The statistical difference of $2.3 \%$ in prepubertal girls is within the standard error of the body fat estimate from skinfold thickness, and is barely biologically meaningful.

Recently we published a study in which prediction formulas for the body composition from body impedance in children were presented (Deurenberg et al. 1989 b). In prepubertal boys and girls percentage body fat could be predicted with an error of about $4 \cdot 2 \%$, which is comparable to the prediction error for the assessment of the percentage body fat from skinfold thickness found in the present study.

In both boys and girls the body mass index gradually increased with age (Tables 1 and 2). As a consequence cut-off points for body mass index as an indicator of overweight or obesity are age-dependent. The correlation of percentage body fat with body mass index 
was lower than the correlation of percentage body fat with skinfold thicknesses (results not shown). This means that in children body mass index is less suitable as a predictor of body fat than skinfold thicknesses. In the literature, however, age-dependent reference values have been published for body mass index as an indicator of obesity in children (RollandCachera et al. 1982).

Tables 7 and 9 show that prepubertal boys with the same amount of subcutaneous body fat (skinfold thickness) have more total fat, i.e. internal fat, than pubertal and post-pubertal boys. In girls the amount of internal fat for the same amount of subcutaneous fat also decreases from prepubertal to pubertal age, and decreases also from pubertal to postpubertal age in the leaner (body fat $<21 \%$ ) subjects. However, in girls with a higher total percentage body fat, the amount of internal body fat is increased (Tables 8 and 10). In the present population such subjects were among older girls (mean (SE) age of girls with body fat $<21 \% 16.7(0.4)$ years, $>21 \% 17.8(0.5)$ years). During adulthood, at the same level of subcutaneous fat, the amount of total fat, i.e. internal body fat, increases again, both in men and in women, as has been shown by e.g. Durnin \& Womersley (1974). The increase in the relative amount of internal fat in the fatter (older) girls can thus be explained by the fact that in general girls reach the adult maturation level at younger ages than boys.

The waist:hips ratio in the present young population was lower at older ages, and this was more pronounced in girls than in boys. A similar observation has been reported by Gillum (1987) and Weststrate et al. 1989. Only in older boys and girls was the waist:hips ratio correlated with measurements of body fat (skinfolds) and only in the older boys and girls was the waist hips ratio correlated with another measure of body fat distribution, trunk: total skinfold ratio (Table 6). This is in accordance with findings of an earlier study (Weststrate et al. 1989) and with findings published by Gillum (1987). As we concluded previously (Weststrate et al. 1989), it seems unlikely that at younger ages a high waist:hips ratio reflects a relatively large (intra) abdominal fat depot, as is the case in adults (Ashwell et al. 1985; Seidell et al. 1987; Weits et al. 1988). The increase in the trunk: total skinfold ratio with age, as found in the present study, confirms the results of an anthropometric study in 2285 Dutch children aged 0-18 years (Weststrate et al. 1989). It indicates that with increasing age, especially in boys, the subcutaneous body fat becomes more centripetally located. However, no skinfolds were measured below the iliac crest level, and it is known that especially in women the gluteal femoral region can be an important fat depot.

In summary, prediction of body density and percentage body fat from skinfold thicknesses is an acceptable method for the assessment of body composition in childhood and adolescence. The waist hips ratio does not appear to be a variable which reflects body fat distribution in young children, but in adolescence the waist:hips ratio becomes meaningful as an indicator of body fat distribution.

\section{REFERENCES}

Ashwell, M., Cole, T. J. \& Dixon, A. K, (1985). Obesity, new insights into the anthropometric classification of fat distribution shown by computed tomography. British Medical Journal 290, 1692-1694.

Boileau, R. A., Lohman, T. G. \& Slaughter, M. H. (1985). Exercise and body composition in children and youth. Scandinavian Journal of Sports Sciences 7, 17-27.

Boileau, R. A., Lohman, T. G., Slaughter, M. H., Ball, T. E., Going, S. B. \& Hendrix, M. K. (1984). Hydration of the fat-free body in children during maturation. Human Biology 56, 651-666.

Boileau, R. A., Wilmore, J. H., Lohman, T. G., Slaughter, M. H. \& Riner, W. F. (1981). Estimation of body density from skinfold thicknesses, body circumferences and skeletal widths in boys and girls aged 8-11 years: comparison of two samples. Human Biology 53, 575-592.

Deurenberg, P., van der Kooy, K., Hulshof, T. \& Evers, P. (1989a). The body mass index as a measure of body fatness in the elderly. European Journal of Clinical Nutrition 43, 231-236

Deurenberg, P., van der Kooy, K., Paling, A. \& Withagen, A. (1989 b). The assessment of the body composition in 8-11 year-old children by bio-electrical impedance. European Journal of Clinical Nutrition 43, 623-629. 
Durnin, J. V. G. A. \& Womersley, J. (1974). Body fat assessment from total body density and its estimation from skinfold thickness: measurements on 481 men and women aged 16-72 years. British Journal of Nutrition 32, 77-97.

Fomon, S. J., Haschke, F., Ziegler, E, E. \& Nelson, S. E. (1982). Body composition of reference children from birth to age 10 years. American Journal of Clinical Nutrition 35, 1169-1175.

Forbes, G. B. (1987). Human Body Composition. Berlin: Springer Verlag.

Forbes, R. M., Cooper, A. R. \& Mitchell, H. H. (1953). The composition of the adult human body as determined by chemical analyses. Journal of Biological Chemistry 203, 359-366.

Forbes, R. M., Mitchell, H. H. \& Cooper, A. R. (1956). Further studies on the gross composition and mineral elements of the human body. Journal of Biological Chemistry 233, 969-975.

Frisancho, A. R. (1981). New norms of upper limb fat and muscle areas for assessment of nutritional status. American Joumal of Clinical Nutrition 34, 2540-2545.

Gillum, R. F. (1987). The association of the ratio of waist to hip girth with blood pressure, serum cholesterol and serum uric acid in children and youths aged 6-17 years. Journal of Chronic Diseases 40, 413-420.

Lohman, T. G. (1986). Applicability of body composition techniques and constants for children and youths. Exercise Sports Sciences Reviews 14, 325-357.

Lohman, T. G., Slaughter, M. H., Boileau, R. A., Bunt, J. \& Lussier, L. (1984). Bone mineral measurements and their relation to bone density in children, youths and adults. Human Biology 56, 667-679.

Lukaski, H. C. (1987). Methods for the assessment of human body composition: traditional and new. American Journal of Clinical Nutrition 46, 537-556.

Mendez, J. \& Lukaski, H. C. (1981). Variability of body density in ambulatory subjects measured at different days. American Journal of Clinical Nutrition 34, 78-81.

Mitchell, H. H., Hamilton, T. S., Steggarda, F. R. \& Bean, H. W. (1945). The chemical composition of the adult human body and its bearing on the biochemistry of growth. Journal of Biological Chemistry 158, $625-637$.

Roede, M. J. \& van Wieringen, J. C. (1985). Growth diagrams 1980, Netherlands third nationwide survey. Tijdschrift Sociale Gezondheidszorg 63, Suppl. 1-34.

Rolland-Cachera, M. F., Sempé, F., Guillod-Bataille, M. M., Patois, E., Pequignot-Guggenbuhl, F. \& Fautrad, V. (1982). Adiposity indices in children. American Journal of Clinical Nutrition 36, 178-184.

Seidell, J. C., Oosterlee, A., Thyssen, M. O. A., Burema, J., Deurenberg, P., Hautvast, J. G. A. J. \& Ruys, J. H. J. (1987). Assessment of intra-abdominal and subcutaneous abdominal fat: relation between anthropometry and computed tomography. American Journal of Clinical Nutrition 45, 7-13.

Siri, W. E. (1961). Body composition from fluid spaces and density: analysis of methods. In Techniques for Measuring Body Composition, pp. 223-244, [J. Brozek and A. Henschel, editors]. Washington, DC: National Academy of Sciences.

Slaughter, M. H., Lohman, T. G., Boileau, R. A., Horswill, C. A., Stillman, R. J., van Loan, M. D. \& Bemben, D. A. (1988). Skinfold equations for estimation of body fatness in children and youths. Human Biology 60, $709-723$.

SPSS (1988). Base Manual SPSS/PC +V2.0. Chicago, Illinois: SPSS Corp.

Tanner, J. M. (1962). Growth and Adolescence. Oxford: Blackwell Scientific Publications.

Van Wieringen, J. C., Wafelbakker, F. \& Verbrugge, H. B. (1968). Groeidiagrammen Nederland. Groningen, The Netherlands: Wolters-Noordhoff.

Weits, T., van der Beek, E. J., Wedel, M. \& ter Haar Romeney, B. M. (1988). Computed tomography measurements of abdominal fat depositions in relation to anthropometry. International Journal of Obesity 12 , $217-225$

Weststrate, J. \& Deurenberg, P. (1988). Body composition in children: proposal for a method to calculate body fat percentage from total body density or skinfold thickness measurements. American Journal of Clinical Nutrition 50, 1104-1115.

Weststrate, J., Deurenberg, P. \& van Tinteren, H. (1989). Indices of body fat distribution and adiposity in Dutch children from birth to 18 years of age. International Journal of Obesity 13, 465-478.

Widdowson, E. M., McCance, R. A. \& Spray, C. M. (1951). The chemical composition of the human body. Clinical Science 10, 113-125. 